\title{
The Symptoms of Child Physical Abuse by Frequency and Specificity
}

\section{Yuri Lee ${ }^{1}$, Garam Noh ${ }^{2}$, Alexandra A Barber ${ }^{3}, K_{\text {Katherine Ginna }}{ }^{1}$, Alison P Wilson ${ }^{1}$, John E Tis ${ }^{4}$ and Dennis M Cullinane ${ }^{5 *}$}

${ }^{1}$ Department of Psychology, Georgetown University, Washington, DC, USA

${ }^{2}$ Department of Social Studies, Harvard University, Cambridge, Massachusetts, USA

${ }^{3}$ Pomona College, Claremont, California, USA

${ }^{4}$ Department of Orthopedic Surgery, Johns Hopkins University School of Medicine, Baltimore, Maryland, USA

${ }^{5}$ Biomechanics Laboratory, Deerfield Academy, Deerfield, Massachusetts, USA

\begin{abstract}
The modern medical history of child physical abuse dates back to 1946 with the publication by John Caffey, Multiple Fractures in the Long Bones of Infants Suffering from Chronic Subdural Hematoma. This now-classic paper was the first modern clinical recognition of child physical abuse and laid the cornerstone for all future clinical diagnoses, as well as prevention and prosecution legislation at state and federal levels. Today, the primary literature is replete with descriptions and analyses of the symptoms of child abuse, but the typical focus is on individual symptoms, their frequencies, and how to diagnose them. Despite the obvious clinical and legal advantages, a quantitatively derived global set of child abuse symptoms based upon both frequency and specificity, and resulting constellations has rarely been addressed or applied. The authors present a quantitative synthesis of the primary literature of child physical abuse, characterizing and ranking symptoms by both frequency and specificity, in hopes that it will serve as a useful tool for future diagnoses and interventions.
\end{abstract}

Keywords: Child physical abuse; Symptoms; Intentional trauma; Frequency; Specificity

\section{Introduction}

For children in the United States between the ages of one and four, the three leading causes of death in 2014 in descending order were unintentional injuries, congenital anomalies, and homicide. Similarly, homicide was the number two causes of death for children less than one year old [1]. Clearly, child abuse is perennially a leading cause of morbidity and mortality in young children and infants [2], with more than four children dying in the United States every day as a consequence [3]. Aside from physical trauma, child abuse also results in significant psychological and sociological impacts [4], as well as personal and civic costs [5]. The authors estimated the lifetime cost per victim of nonfatal child maltreatment was $\$ 210,012$ (2010 dollars), with a total lifetime economic burden resulting from all new cases of fatal and nonfatal child maltreatment at approximately $\$ 124$ billion. Clearly, child abuse is a significant social dilemma, and it is manifested in different forms, including neglect, sexual, emotional, and physical, and yet its clinical recognition is relatively recent. Caffey's study garnered a great deal of attention in the medical community because of its revolutionary effort to recognize how patterns of injury, medical history, and parent behavior are indicative of child abuse. In doing so, he was the first to question the sanctity of the parent-child relationship. He was also the first to identify an abuse constellation: long bone fracture and subdural hematoma. A constellation arises when two or more symptoms of abuse are manifested in a potential victim and whose collective suspicion index rises above the sum of the individual symptoms. In this way, Caffey presciently defined the landscape for all future abuse studies.

Despite Caffey's findings, the prevalence of child abuse was not widely acknowledged by the general population until Kempe et al. [6] published their research on the battered-child syndrome, inciting the first national movement towards addressing child abuse. This movement culminated in the passage of the 1974 Child Abuse Prevention and Treatment Act. Despite these advances, it was estimated that 1,580 children in the United States died from physical abuse in 2014 [3], and failure to recognize child maltreatment results in chronic exposure to high-risk environments where re-injury or death may occur, with traumatic brain injury (95\%) and bruising (90\%) the most common injuries [7]. Consequently, accurate diagnoses and timely interventions by healthcare professionals in cases of child abuse are essential. Further, childcare professionals such as daycare providers, school nurses, and even gym teachers can serve as the frontline of detection, and thus their recognition of symptoms would be critical in the early characterization of abuse. Thus, the characterization of child abuse symptoms by numerical frequency and specificity as they are referenced in the primary literature would be a useful tool for clinicians, as well as nonmedical childcare personnel.

\section{Frequency and specificity}

For the purposes of this study, frequency is a simple quantitative representation of the number of appearances of a symptom in relation to NAT, whereas symptoms of high specificity are typically limited to and thus specific to NAT, and rare in cases of accidental trauma (AT). Thus, they are highly specific to NAT, but also occur rarely in AT. In contrast, highly frequent NAT symptoms may also be frequent in AT. Thus, scapular fractures, for example, are relatively rare in AT, but highly specific for NAT, because biomechanically, they are much less likely to occur in more typical childhood accidents. In contrast, long bone fractures are frequent in NAT, but not highly specific, as they occur frequently in AT, as well.

Although NAT occurs in children of all ages, it is typically those younger than three that are victims of abuse [8]. Younger victims of

*Corresponding author: Dennis M Cullinane, Biomechanics Laboratory, Deerfield Academy, Deerfield, MA 01342, USA, Tel: 978-463-0532; E-mail: dcullinane@ deerfield.edu

Received October 06, 2017; Accepted November 21, 2017; Published November 29, 2017

Citation: Lee Y, Noh G, Barber AA, Ginna K, Wilson AP, et al., (2017) The Symptoms of Child Physical Abuse by Frequency and Specificity. J Forensic Biomed 8: 136. doi: 10.4172/2090-2697.1000136

Copyright: (c) 2017 Lee Y, et al. This is an open-access article distributed under the terms of the Creative Commons Attribution License, which permits unrestricted use, distribution, and reproduction in any medium, provided the original author and source are credited. 
child abuse are less capable of resisting, escaping, or voicing accusations against their abusers, and have more gracile and mechanically susceptible skeletons [9]. Thus, emergency department (ED) personnel need to be able to identify strongly suggestive constellations, or groupings of symptoms that form a clearly recognizable pattern for child abuse [10].

\section{Constellations of abuse}

Like constellations, or clusters of stars in the night sky that together tell a story to the stargazer, single symptoms of abuse can form clusters, or constellations, that tell a story to a caregiver. The concept of a constellation of child abuse incorporates multiple symptoms whose individual frequencies combine in a manner greater than the sum of the individual symptoms. For example, a highly indicative constellation for child abuse would include: subdural hematoma, an undocumented healed fracture, defensive bruises on the forearms, and posterior rib fractures. Each of these injuries is itself a symptom of child abuse, but when found in a collective constellation, their individual suspicion indices create a non-linear, highly positive likelihood. Additionally, barring extremely rare incidences, this constellation of symptoms does not suggest a single accidental traumatic event such as tripping and falling forward onto the ground while running. In contrast, a nonindicative constellation would more likely include bilateral abrasions on the knees, a distal radial fracture, and bilateral abrasion on the palms of the hands. The latter example suggests a single traumatic event and AT symptoms (a fall forward to the ground). These two intentionally illustrative examples easily reflect NAT and AT, respectively, however, the reality is typically not as clear. Thus, in order to characterize child abuse constellations and evaluate abuse likelihoods, professionals are in need of a definitive ordering of symptoms of NAT in terms of both frequency and specificity. If medical professionals evaluate victims of child abuse with an understanding of symptom frequency, specificity, and resulting constellations, they would likely have more confidence in those evaluations, and their diagnoses would be more accurate. And ultimately, young patients can receive the medical care, legal support, and social care they need; even in the face of uncooperative guardians who often try to deny the possibility of abuse out of fear or guilt, and particularly when they are the perpetrators [10].

The objective of this global analysis of child abuse symptoms is to mine the primary literature of child abuse in order to generate hierarchical tables of both abuse symptom frequency, and specificity. It is hoped these tables might then be available to medical and other childcare personnel to aid in identifying abuse symptoms and constellations. Ultimately, trained medical clinicians are responsible for the diagnosis of child abuse or maltreatment. However, equipping them and frontline childcare providers with more powerful diagnostic tools will only serve to increase child safety. Of course, protocols for the reporting of these symptoms on all levels need to be in place so that ultimately, cases end up in the hands of trained medical and child advocacy professionals.

\section{Materials and Methods}

The focus of this analysis, congruent with analytical trends in the primary literature, is on children up to 5 years of age. Symptoms of NAT were assessed in terms of both their frequency and specificity. A total of 111 studies of physical symptoms of child abuse mined from Public Medline using the key terms: child abuse (symptoms), child maltreatment (symptoms), child non-accidental trauma (symptoms), child trauma (symptoms), pediatric abuse (symptoms), child abuse symptom frequency, physical abuse children, and child abuse symptom specificity. Studies were examined for references to the frequency and/ or specificity of any injuries associated with physical abuse. Studies were deemed appropriate for use based on three basic criteria: injuries of child abuse had to be the central focus of the article, authors had to make clear indications of trauma as non-accidental (NAT) versus accidental (AT), and there had to be at least one reference to the frequency (percentage of cases exhibiting a symptom) and/or specificity (the authors' subjective view of how closely tied a symptom is to NAT) of a symptom of child abuse. If these three requirements were not met, that study was excluded. Each statistic regarding child abuse symptoms (e.g. retinal hemorrhage) was recorded on a Microsoft Excel ${ }^{\circ}$ spreadsheet with the physical symptoms listed by author(s) and publication. Two tables were created; one dedicated to symptom frequency and the other to symptom specificity.

After the frequency data was compiled, a mean frequency was calculated for each injury associated with child abuse and an ordering of frequency was created- from most frequent to least frequent. For studies providing a percentage range for frequency (i.e., 10-25\%), the mean of the range was calculated (17.5\%) and used to represent that data. This final frequency value for a symptom was calculated by averaging the frequency statistics provided by all authors into a final mean value of frequency for that symptom. It is important to note that individual studies were not weighted for study size.

The data for the specificity chart was collected in a two-step process: all references to a symptom being "specific" for child abuse were noted in the Microsoft Excel ${ }^{\circ}$ spreadsheet with a scale containing a numerical range from 0 to 4 (lowest specificity to highest specificity, respectively) and all language used by authors to qualify the specificity (mentions of high or low specificity of symptoms) was recorded next to the numerical values on the table. For example, a score of 4 was given to the term "highest specificity", whereas a 1 was awarded to a symptom regarded as "low specificity". The number of studies referring to a symptom as "specific", for example, we took to reflect the degree of consensus within the scientific/medical community. Average specificity values were calculated for each symptom based on this scale (Table 1).

For example, metaphyseal lesions were mentioned as being specific to child abuse in eleven different articles. One of these articles described it as a specific injury ( 2 points), with no further qualifier, while the other ten articles stated that it was a highly specific injury ( 3 points). As a result, metaphyseal lesions were ascribed a total point value of 32 points from these eleven articles, leading to an average specificity value of 2.9. This numerical specificity value was then compared to the values for other symptoms in order to formulate a hierarchy of specificity. Furthermore, if two injuries received the same average specificity value, the symptom supported by more studies was given priority over the other in the ordering. Regardless, they received appropriately identical scores.

\section{Results}

The data derived from all 111 studies reviewed was compiled into frequency and specificity (Tables 2 and 3 ) and divided further into four levels of severity based on natural breaks in the data. The frequency dataset was divided into Levels 1-4 based on the percentage of abused children who suffered the injury. The specificity data was divided into Levels 1-4 as well, whose rankings were designated based on the specificity values calculated as shown in Table 1, with the four levels of each table designated by colors, with blue representing level one (the least specific or least frequent injuries), and increasing in intensity with yellow, orange, and red, representing increasing levels of specificity or frequency, respectively. 
Citation: Lee Y, Noh G, Barber AA, Ginna K, Wilson AP, et al. (2017) The Symptoms of Child Physical Abuse by Frequency and Specificity. J Forensic Biomed 8: 136. doi: 10.4172/2090-2697.1000136

Page 3 of 6

\begin{tabular}{|c|c|}
\hline Specificity Score & Specificity Terms \\
\hline 4 & Highest specificity, extremely specific, most specific, strongest specificity, other related terms \\
\hline 3 & Highly specific, strongly specific, strongly suspicious, strong predictor, significant specificity, other related terms \\
\hline 2 & Specific, suspicious, suggestive, moderately specificity, other related terms \\
\hline 1 & Low specificity, modest specificity, occasionally specific, other related terms \\
\hline
\end{tabular}

Table 1: Specificity level descriptors table.

\begin{tabular}{|c|c|c|c|}
\hline Level 1 & Level 2 & Level 3 & Level 4 \\
\hline Intraperenchymal hemorrhage $7.2 \%$ & Femoral fx $22.5 \%$ & Long bone fx $49.8 \%$ & Soft tissue injury $74.0 \%$ \\
\hline Bites $6.9 \%$ & Subarachnoid hemorrhage $21.0 \%$ & Bruising $49 \%$ & Subdural hematoma $55.4 \%$ \\
\hline Abrasion $5.7 \%$ & Rib fx $19.0 \%$ & Cerebral atrophy $45.0 \%$ & Multiple fx $51.3 \%$ \\
\hline Temporal fx $5.7 \%$ & Petechia $15.9 \%$ & Subdural hygroma $45.0 \%$ & Transverse fx $50.3 \%$ \\
\hline Frontal fx $4.3 \%$ & Head trauma $15.0 \%$ & Ex vacuo vernticulomegaly $45.0 \%$ & Single fx $50.0 \%$ \\
\hline Multiple skull fx $4.3 \%$ & Diffuse brain edema/axonal injury $14.0 \%$ & $\begin{array}{l}\text { Homogeneous hyperdense subdural } \\
\text { hematoma } 33.0 \%\end{array}$ & Fx in various stages $50 \%$ \\
\hline Spine/pelvis/extremity fx $4.1 \%$ & Retinal hemorrhage $13.4 \%$ & Metaphyseal injury $31.3 \%$ & \\
\hline Clavicular fx $3.6 \%$ & Parietal fx $13.1 \%$ & Brain injury $29.7 \%$ & \\
\hline Epidural hematoma 3.0\% & Radius/ulna fx $13.1 \%$ & Humeral fx $29.0 \%$ & \\
\hline Orbital fx $2.9 \%$ & Abdominal/thoracic trauma $12.2 \%$ & Skull fx $28.6 \%$ & \\
\hline Spinal fx $2.0 \%$ & Tibia/fibula fx $12.1 \%$ & General fx $28.0 \%$ & \\
\hline Spinal injury $1.6 \%$ & Burns $11.0 \%$ & & \\
\hline \multicolumn{4}{|l|}{ Foot fx $1.2 \%$} \\
\hline \multicolumn{4}{|l|}{ Hand fx $0.4 \%$} \\
\hline Occipital fx $0.1 \%$ & & & \\
\hline
\end{tabular}

Table 2: Child /abuse symptoms by frequency.

\begin{tabular}{|c|c|c|c|}
\hline Level 1 & Level 2 & Level 3 & Level 4 \\
\hline Epidural hematoma $0(1)$ & Skull fx 1.7(6) & Metaphyseal lesion/fx 2.9 (21) & Absent/evolving trauma $3.5(2)$ \\
\hline \multirow[t]{23}{*}{ Nondisplaced spiral diaphyseal fx $0(1)$} & Clavicular fx 1.6(8) & Rib fx 2.8(31) & Retinal hemorrhage $3.3(3)$ \\
\hline & Long bone fx 1.5(2) & Multiple fx $2.7(14)$ & Spiral fx humerus $3.3(3)$ \\
\hline & Linear skull fracture $1(4)$ & Humeral fx 2.5(8) & Scapular fx 3 (12) \\
\hline & Subperiosteal new bone $1(1)$ & Fx in different stages $2.5(4)$ & Sternal fx $3(6)$ \\
\hline & Transverse long bone fx $1(1)$ & Vertebral fx 2.5(1) & Metaphyseal injury/lesions 3(3) \\
\hline & Multiple skull fx 1(1) & Complex skull fx $2.5(4)$ & Spinous process $\mathrm{fx} 3(3)$ \\
\hline & Parietal fx 1(1) & Unilateral ear bruising $2.5(2)$ & Bilateral fx 3(3) \\
\hline & Diaphyseal fx 1(1) & Femoral fx 2.4(9) & Subdural hematoma 3(2) \\
\hline & Single $f \times 1(1)$ & Tibia/fibula fx 2.3(3) & Epiphyseal fx 3(2) \\
\hline & Supracondylar humeral fx 1(1) & Metaphyseal-epiphyseal fx 2(5) & Avulsion fx acromion process $3(1)$ \\
\hline & Mixed density DSH 1(1) & Long bone fx 2(4) & Pelvic 3(1) \\
\hline & Homogeneous hyperdense SDH 1(1) & Tibia $\mathrm{fx} 2(4)$ & Hypodense SDH 3(1) \\
\hline & Diffues axonal injury $1(1)$ & Sternal fx 2(3) & Proximal tibial CNL 3(1) \\
\hline & Cerebral edema $1(1)$ & Fibula fx 2(2) & Prior history of trauma $3(1)$ \\
\hline & & Epiphyseal separation 2(2) & Soft tissue injury $3(1)$ \\
\hline & & Bruising neck 2(2) & Spinal injury 3(1) \\
\hline & & Digital fx 2(2) & Hemorrhagic retinopathy $3(1)$ \\
\hline & & Bruising 2(1) & \\
\hline & & Bruising head 2(1) & \\
\hline & & Bruising buttocks 2(1) & \\
\hline & & Bruising perineum 2(1) & \\
\hline & & Bruising genitalia 2(1) & \\
\hline & & Bruising trunk 2(1) & \\
\hline
\end{tabular}

Table 3: Child abuse symptoms by specificity.

\section{Discussion and Conclusion}

Certain symptoms were found in an overwhelming proportion of abused children, including: soft tissue injuries (74\%), subdural hematoma $(55.4 \%)$, multiple fractures $(51.3 \%)$, transverse fractures
(50.25), and fractures in various stages (50\%). Interestingly, the highest specificity score went to absent or evolving trauma (3.5). These are injuries without corresponding trauma events, or injuries whose stated cause(s) change over time, or injuries that themselves continue to evolve over time. Kellogg [11] and Kemp et al. [12] confirm that these 
circumstances can be consistent to highly indicative of intentional trauma. This symptom is unique in focusing more on the interview and interaction with the patient or guardian, but encompassed the concept of physical trauma, and so was included. Following this were retinal hemorrhages (3.3), scapular fractures (3.0) and sternal fractures (3.0), all less likely to happen by common accidental trauma mechanics. A general note must be made about redundant symptoms such as various forms of single fracture. When possible, redundancies were incorporated into a common pool, averaging their scores appropriate to number of times referenced. Given that, because the terminology in the primary literature was sometimes very precise, there were occasionally symptoms that seemingly overlapped a great deal, such as single fracture and transverse fracture, but they were treated separately because of the detailed term, transverse, for example, implying a specific mechanical environment.

Some symptoms were highly ranked on one list but scored relatively low on the other. For example, a single fracture is highly frequent ( 1 in 2 abused children), putting it at Level 4 for frequency, but is unspecific to child abuse (a score of 1 at the bottom of Level 2). Transverse fractures are also very common (evident in just over 1 in 2 abused children), but relatively unspecific (Level 2 specificity). In contrast, there were also symptoms found to be relatively infrequent, but highly specific. Although these injuries may not be encountered often in evaluating children for child abuse, when they do appear in a patient, they should raise suspicion. For example, spinal injury is experienced by just over $6 \%$ of abused children, but rated Level 4 for specificity. Most importantly however, some symptoms were ranked relatively highly in both frequency and specificity. These include soft tissue injuries (74\% and a specificity score of 3 , some form of subdural hematoma (55.4\% and a specificity score of 3$)$, and fractures ( $50 \%$ and specificity score of 3). The magnitude of the importance of soft tissue injuries in terms of specificity is highlighted by the work of McMahon et al. [13], who reported more than 3 million cases of child abuse in a single year that included cutaneous symptoms, making it the most recognizable symptom of abuse. Jackson et al. [14] highlight the contribution to delay in abuse diagnoses via clinical inattention to soft tissue findings. Accordingly, soft tissue bruising, for example, the result capillary failure via blunt trauma [15-17], in any of the locations indicated by the Frequency or Specificity (Tables 2 and 3) should also be considered a significant finding.

When considering the symptoms of child abuse, it must be recognized that some mechanical interaction necessarily occurred between a victim and a perpetrator, or some object utilized by the perpetrator. If multiple symptoms exist but do not share an anatomical location or even body region (posterior ribs and metaphyseal lesion), it is more likely that multiple trauma events occurred (as stated above, multiple events of the same trauma can also occur). This is the heart of the multiplicative power of a constellation of abuse: Multiple injuries of even mid or low-level suspicion, when combined, will increase the index of suspicion by a factor greater than their individual values because they can be attributed to multiple traumas and an environment of continuing abuse. Thus, multiple mechanical insults create multiple symptoms, and are generated because the maltreatment environments that cause them tend to involve chronic physical abuse.

It is also important to note at this point that weighting for study sizes was considered but not utilized, as ultimately, the choice of studying any one particular symptom is essentially a random process and weighting of larger studies puts those particular symptoms in the fore, with no regard to symptom importance. This is especially so with regard to specificity, but even when considering frequency, the residual effect of according any kind of weight to larger data studies necessarily biases the reader's sense of inter-symptom importance simply because an author or set of authors chose to investigate any particular symptom(s). Accordingly, the authors felt that the relationship between specificity and frequency, and the magnitude of the sensitivity of the two tables was better served without weighting.

The frequency and specificity tables generated in this study are meant to be useful diagnostic tools, and beyond those, the power of derived symptom constellations cannot be overstated. The combination of multiple symptoms in a highly indicative constellation can be more strongly suggestive of child abuse than the presence of any one, more noteworthy symptom, either for frequency or specificity. The ordering of child physical abuse symptoms by frequency and specificity can provide guidelines for medical personnel to be able to more assuredly distinguish accidental trauma from non-accidental trauma in children, and to construct diagnostic constellations. These results suggest that symptoms (and especially multiple symptoms) should be followed up with further investigation; the vigor of which should be relative to their frequencies and specificities. Additionally, as indicated in the specificity scale, absent or evolving trauma is highly indicative of abuse, as should be any symptom that is not biomechanically likely, motor skillsappropriate, or temporally consistent with the stated etiology of injury presented by the child or caretaker.

Finally, and as stated earlier, in 2014, homicide was the third leading cause of death for children age 1-4, and of children age $<1$, combined homicide numbers similarly rank it as the second most common cause of injury death. Considering the potential for missed diagnoses, these numbers may be even larger. Today, 54 years since Kempe's Battered Child and 70 years since Caffey's groundbreaking treatise, a clear public health crisis of child abuse still exists. Sadly, diagnostic errors [18] and systematic under-identification [19] and underreporting of cases of abuse $[20,21]$ remain prevalent. Alarmingly, this is true even when the level of suspicion is high and the decision maker is a trained medical professional [22]. Further concerning is the unnecessary gap in the tools available to medical, paramedical, and childcare professionals, with a need for more navigable and "user-friendly" screening tools [19]. Nonaccidental trauma often presents as a broad spectrum of symptoms that might superficially seem unimportant, but are actually "sentinel" injuries [23], whose collective constellations should draw intense caregiver scrutiny. Thus, more work needs to be done in educating and arming healthcare and childcare professionals so that they are better equipped and more confident in their roles characterizing, raising suspicion of, and reporting abuse.

\section{References}

1. https://www.cdc.gov/injury/wisqars/leadingcauses.html

2. Pierce MC, Bertocci G (2008) Injury biomechanics and child abuse. Ann Rev Biomed Eng 10: 85-106.

3. https://www.acf.hhs.gov/sites/default/files/cb/cm2014.pdf

4. Johnson JG, Cohen P, Brown J, Smailes EM, Bernstein DP (1999) Childhood maltreatment increases risk for personality disorders during early adulthood. Arch Gen Psychiatry 56: 600-606.

5. Fang X, Brown DS, Florence CS, Mercy JA (2011) The economic burden of child maltreatment in the United States and implications for prevention. Child Abuse and Neglect 36: 156-165.

6. Kempe CH, Silverman FN, Steele BF, Droegemuller W, Silver HK (1962) The battered child syndrome. J Am Med Assoc 181: 17-24.

7. Pierce MC, Kaczor K, Acker D, Webb T, Brenzel A (2017) History, injury, and psychosocial risk factor commonalities among cases of fatal and near-fatal 
Citation: Lee Y, Noh G, Barber AA, Ginna K, Wilson AP, et al. (2017) The Symptoms of Child Physical Abuse by Frequency and Specificity. J Forensic Biomed 8: 136. doi: 10.4172/2090-2697.1000136

Page 5 of 6

physical child abuse. Child Abuse and Neglect 69: 263-277.

8. Maguire S (2010) Which injuries may indicate child abuse? ADC Education and Practice Edition 95: 170-177.

9. Cullinane DM, Einhorn TA (2002) The biomechanics of bone. In: Bilezekian JP, Raisz LG, Rodan GA (eds.), Principles of the Biology of Bone (2nd edn.). Academic Press, UK. pp: 1-15.

10. http://ota.org/media/34582/p02_abuse-revision.ppt

11. Kellogg ND (2017) Evaluation of suspected child physical abuse. Pediatrics 119: $1232-1241$.

12. Kemp AM, Dunstan F, Harrison S, Morris S, Mann M (2008) Patterns of skeletal fractures in child abuse: A systematic review. BMJ 337: a1518.

13. McMahon P, Grossman W, Gaffney M, Stanitski C (1995) Soft tissue injury as an indication of child abuse. J Bone Joint Surg 77: 1179-1183.

14. Jackson AM, Deye KP, Halley T, Hinds T, Rosenthal E (2015) Curiosity and critical thinking: Identifying child abuse before it is too late. Clin Ped 54: 54-61.

15. Huang L, Bakker N, Kim J, Marston J, Grosse I (2012) A multi-scale finite element model of bruising in soft tissues. J Forensic Biomech 3: 1-5.

16. Tang K, Sharpe W, Schulz A, Tam E, Grosse I (2013) Determining bruise etiology in muscle tissue using finite element analysis. J Forensic Sci 59: 371 374

17. Grosse IR, Huang L, Davis J, Cullinane DM (2014) A multilevel hierarchical finite element model for capillary failure in soft tissue. J Biomech Eng 136: 1-8.

18. Anderst J, Nielsen-Parker M, Moffatt M, Frazier T, Kennedy C (2016) Using simulation to identify sources of medical diagnostic error in child physical abuse. Child Abuse Negl 52: 62-69.

19. Hoft M, Haddad L (2017) Screening children for abuse and neglect: A review of the literature. J Forensic Nursing 13: 26-34.

20. Flaherty EG, Sege RD, Hurley TP (2008) Translating child abuse research into action. Pediatrics 122: S1-S5.

21. Leeb RT, Fluke JD (2015) Child maltreatment surveillance: enumeration monitoring, evaluation and insight. Health Promot Chronic Dis Prev Can 35 138-140.

22. Flaherty EG, Sege RD, Griffith J, Price LL, Wasserman R (2008) From suspicion of physical child abuse to reporting: primary care clinician decisionmaking. Pediatrics 122: 611-619.

23. Tiyyagura G, Beucher M, Bechtel K, Pade KH (2017) Nonaccidental injury in pediatric patients: Detection, evaluation, and treatment. Pediatr Emerg Med Pract 14: S1-S2 